\title{
Inversión extranjera directa, movilidad laboral y derrames de conocimiento en Costa Rica
}

\author{
Foreign direct investment, labor mobility \\ and knowledge spillovers in Costa Rica
}

Ricardo Monge González'

Juan Carlos Leiva Bonilla

Juan Antonio Rodríguez Álvarez ${ }^{3}$

Fecha de recepción: 20 de enero del 2012 Fecha de aprobación: 19 de marzo del 2012

Monge, R; Leiva, J; Rodríguez, J. Inversión extranjera directa, movilidad laboral y derrames de conocimiento en Costa Rica. Tecnología en Marcha. Vol. 25, № 5. Pág I03-I I 5.

Este artículo cuenta con el aval de la

Vicerrectoría de Investigación y Extensión del Tecnológico de Costa Rica
Economista. Escuela de Administración de Empresas, Tecnológico de Costa Rica Teléfonos: 2253-8828 / 2253-3324. Correo electrónico: rmonge@caatec.org

2 Administrador de Empresas. Escuela de Administración de Empresas, Tecnológico de Costa Rica. Teléfonos: 2550-9052 / 8844-8 |80. Correo electrónico: jleiva@itcr.ac.cr

3 Estadístico y administrador de negocios. Escuela de Administración de Empresas, Tecnológico de Costa Rica. Teléfono: 2250-7567. Correo electrónico: jaral07@gmail.com 


\section{Resumen}

Este trabajo busca determinar la existencia e importancia de los derrames de conocimiento asociados con la movilidad laboral desde las multinacionales (MNCS) que operan bajo el régimen de zonas francas (ZF) en Costa Rica; es decir, el impacto de tales derrames de conocimiento sobre el desempeño de las empresas locales que contratan ex empleados de MNCS. Se observó que, de un total de 4I I 49 empleados de MNCS que dejaron de laborar para estas empresas entre el 200 I y el 2007, un tercio se trasladó a empresas locales ( 15 139).

El impacto de los derrames de conocimiento sobre el desempeño de empresas locales se midió en términos de tres variables: ventas, empleo y productividad media del trabajo; para esto se empleó un panel de empresas locales para el periodo que va del 2007 al 2009. Mediante el empleo de modelos econométricos sugeridos por la literatura en este campo, se encontró evidencia de que las empresas locales que han contratado ex empleados de MNCS obtienen un mejor desempeño en términos de crecimiento de sus ventas y empleo, que aquellas empresas locales que no contratan ex empleados de MNCS. Es decir, se obtuvo una clara evidencia de la externalidad positiva asociada con los derrames de conocimiento para Costa Rica, producto de la movilidad laboral desde las MNCS. No se encontró esta evidencia cuando el desempeño de las empresas locales se mide en términos de la productividad media del trabajador.

Finalmente, los resultados señalan la importancia de llevar a cabo nuevas investigaciones sobre la capacidad de absorción de las empresas locales para potenciar más los impactos positivos de los derrames de conocimiento, así como respecto al impacto de tales derrames sobre la productividad total de los factores.

\section{Palabras clave}

Conocimiento, derrames, desempeño,externalidades, inversión extranjera directa, multinacionales, movilidad laboral.

\begin{abstract}
This paper aims to determine the existence of knowledge spillovers associated to labor mobility from multinational companies (MNCS) operating in Costa Rica. That is, the impact of such knowledge spillovers over the performance of local firms that hire MNCS' former employees. We found that, out of a total of 4I I 49 MNCS' employees who quitted working for these companies between 200I and 2007, inclusive, one third shifted to work for local firms ( 15 |39). Knowledge spillovers' impact on the latter was measured in terms of three variables: sales, employment and average labor productivity, using a panel of local firms for the period between 2007 and 2009, inclusive. Using econometric models suggested by the literature on this field, we found evidence indicating that local firms which hired MNCS' former employees perform better in terms of employment and sales growth that those local firms not hiring MNCS' former employees. We did not find the same evidence when measuring local firms' performance using average labor productivity. Finally, results point out the importance of conducting new research about the local firms' absorption capacity in order to boost even more the positive impact of knowledge spillovers, as well as the impact of such spillovers on total factor productivity.
\end{abstract}

\section{Key words}

Knowledge spillovers, performance, externalities, multinational companies, labor mobility. 


\section{Introducción}

Las empresas multinacionales (MNCS), en especial aquellas de alta tecnología, tienden a caracterizarse por poseer el conocimiento más avanzado en su área a nivel mundial. Debido a esto, se tiende a argumentar que las MNCS disfrutan de una superioridad tecnológica y fuertes habilidades administrativas, las cuales pueden ser transferidas o imitadas por empresas locales del país anfitrión, especialmente en el caso de países en vías de desarrollo. Este fenómeno es conocido como derrames de conocimiento (knowledge spillovers) y se definen como externalidades positivas que benefician a las empresas locales gracias a la presencia de inversión extranjera directa (IED), la cual puede resultar en incrementos de la productividad en estas empresas (Blomström, 1986; Caves, 1974 \& Spencer, 2008).

Previos estudios sobre esta materia han producido resultados mixtos o contradictorios respecto a los derrames de conocimiento asociados con la IED en países en vías de desarrollo o emergentes. Algunos estudios han encontrado evidencia de efectos positivos de estos derrames en las empresas locales (Blomström, 1986; Buckley, Clegg \& Wang, 2007; Tian, 2007; Wei \& Liu, 2006), mientras que otros estudios, por el contrario, han encontrado que la IED no ha producido derrames de conocimiento alguno, o bien, que sus efectos han sido negativos para las empresas locales (Feinberg \& Majumdar, 200 I).

Según Zhang, Li, Li \& Zhou (20l0), existen dos razones del por qué se dan estos contradictorios resultados. En primer lugar, el enfoque adoptado por los estudios empíricos sobre los derrames de conocimiento asociados a la IED se orienta en el hecho de si la presencia de IED afecta la productividad de las empresas locales. En palabras de Görg y Strobl (200I), una de las limitantes de los estudios antes citados es que tratan los mecanismos específicos por los que, se supone, suceden los derrames de conocimiento, como una especie de "caja negra". En segundo lugar, los derrames de conocimiento involucran un proceso por medio del cual las empresas locales aprenden de las empresas extranjeras.

Así, los efectos de los derrames de conocimiento asociados con la IED también dependen del papel que juegan las empresas locales como receptoras de estos derrames. En otras palabras, es necesario tomar en consideración la capacidad de estas empresas para absorber y adaptar conocimientos (capacidad de absorción), ya que de esto dependerá si se producen o no efectos positivos de los derrames de conocimiento (Lim, 200 I).

En un esfuerzo por clarificar el primer problema del enfoque de los estudios señalados, Saggi (2002) identifica tres canales por medio de los cuales pueden ocurrir derrames de conocimiento desde las MNCS hacia las empresas locales: (i) efectos demostración, los cuales incluyen la imitación o ingeniería inversa de los productos o prácticas de las empresas MNCS por parte de las empresas locales, (ii) movilidad laboral, que les permite a los empleados capacitados por MNCS aplicar sus conocimientos en las empresas locales cuando ellos dejan de trabajar en las primeras, y (iii) encadenamientos verticales hacia adelante y hacia atrás entre empresas MNCS y sus proveedores locales. La importancia de este aporte es que, en aquellos estudios donde se aclara el canal por el cual se cree que se producen los derrames de conocimiento, los resultados de las externalidades positivas de tales derrames son muy sólidos.

Al ser Costa Rica uno de los países más exitosos en la atracción de IED durante los últimos veinte años, especialmente en el área de alta tecnología (Monge, 20l0), cabe preguntarse hasta qué punto el desempeño de las empresas costarricenses se ha visto afectado debido a la movilidad laboral desde las MNCS que operan en este país. Responder esta pregunta constituye la razón de ser del presente artículo. Por ello, el objetivo central ha sido identificar y cuantificar la existencia de derrames de conocimiento asociados con la movilidad laboral desde las empresas multinacionales (MNCS) hacia el sector productivo privado costarricense (empresas pequeñas, medianas y grandes) y su impacto sobre el desempeño de estas empresas (ventas, empleo y productividad media del trabajo); todo con el propósito de sugerir recomendaciones de política pública.

Este documento presenta los principales hallazgos de un proyecto de investigación en el campo de los derrames de conocimiento mediante la movilidad laboral en las MNCS que operan en Costa Rica, y fue realizado gracias al patrocinio del Banco Interamericano de Desarrollo (BID), la Comisión Asesora en Alta Tecnología (Caatec) y laVicerrectoría de Investigación delTecnológico de Costa Rica (TEC). 
La realización de este esfuerzo fue posible gracias al suministro de valiosa información por parte de la Coalición Costarricense de Iniciativas de Desarrollo (Cinde), la Caja Costarricense de Seguro Social (CCSS) y la Promotora Costarricense de Comercio Exterior (Procomer), así como la recolección de datos mediante una encuesta al sector productivo costarricense por parte de la empresa Unimer Research Internacional.

El documento está organizado en tres secciones, más la introducción. La primera sección discute la trabajos previos sobre la movilidad laboral desde las MNCS y sus externalidades positivas, la importancia de los flujos de IED atraídos por Costa Rica durante las últimas décadas, bajo el régimen de Zonas Francas (ZF), y la movilidad laboral producida en las empresas MNCS de alta tecnología que han operado en este país entre los años 200 I y 2007. La segunda sección presenta los resultados sobre el impacto de la aplicación de conocimientos adquiridos en estas MNCS sobre el desempeño de las empresas locales costarricenses. La tercera y última sección presenta los principales hallazgos del documento, las recomendaciones de política y algunas futuras líneas de investigación.

Los resultados, interpretaciones y conclusiones expresados en este documento son a criterio de los autores y no reflejan necesariamente los puntos de vista de las empresas, instituciones u organizaciones que apoyaron esta iniciativa; cualquier error $u$ omisión es atribuible únicamente a los autores.

\section{Movilidad laboral desde las multinacionales, inversión extranjera directa y externalidades}

\section{Estado de la cuestión}

La existencia de MNCS operando en un país anfitrión puede facilitar la adquisición de conocimiento por parte de empresas locales, ya sea por medio de acuerdos comerciales entre las MNCS y sus proveedores locales (knowledge transfer), o mediante derrames de conocimiento desde las MNCS hacia las empresas locales. Los derrames de conocimiento son particularmente importantes, ya que su existencia representa una externalidad positiva para el resto de la economía del país anfitrión. A nivel de empresa, un derrame de conocimiento se define como "el conocimiento creado por una empresa multinacional, el cual es utilizado por una empresa local en el país anfitrión, sin que la compañía multinacional reciba alguna compensación" (Javorcik, 2004).

Como se mencionó en la introducción, existen tres canales a través de los cuales los derrames de conocimiento puede ser generados: (i) efectos demostración, (ii) movilidad laboral y (iii) encadenamientos verticales (Saggi, 2002).

Existe poca evidencia sobre el impacto de la movilidad laboral desde las MNCS en las empresas del país anfitrión. Así, en América Latina, Markunsen y Trofimenko (2007) encontraron, en el sector manufacturero de Colombia, impactos positivos de la capacitación brindada por expertos extranjeros a empleados locales que luego se trasladaron a trabajar a empresas domésticas; mientras que Poole (2008) observó externalidades positivas en empresas domésticas brasileñas asociadas con la movilidad de trabajadores desde las MNCS.

Por otra parte, Görg y Strobl (2005) encontraron mayores niveles de productividad en las empresas locales del sector manufacturero de Ghana cuando estas eran creadas por ex empleados de MNCS; por último, Hale y Long (2006) hallaron, en el caso de trabajadores altamente calificados de China, externalidades positivas asociadas con la movilidad laboral desde las MNCS a las empresas locales de este país, siempre que ellas fueran las más avanzadas tecnológicamente.

\section{Inversión extranjera directa y capacitación a trabajadores costarricenses}

Como parte del nuevo modelo de desarrollo basado en la apertura económica desde mediados de la década de los ochenta, Costa Rica inició un proceso de liberalización unilateral de su comercio exterior, el cual ha continuado hasta el presente con diferente intensidad'. Las zonas francas (ZF) surgieron en Costa Rica durante el mismo periodo de la apertura económica como un mecanismo para la atracción de inversión extranjera directa (IED), lo cual ayudó a promover las exportaciones de productos no tradicionales a terceros países, crear nuevas oportunidades de empleo, mejorar la balanza de pagos y apoyar la diversificación de la base productiva nacional.

\footnotetext{
Véase por ejemplo Monge y Lizano (1997).
} 
El régimen de ZF es un conjunto de incentivos y beneficios otorgados por el gobierno costarricense a las empresas (principalmente MNCS) que hacen nuevas inversiones en el país. Un aspecto importante de las ZF es su potencial como fuente generadora de derrames de conocimiento debido a la movilidad laboral desde las MNCS, tema central de la presente esta investigación.

En cuanto a la experiencia de Costa Rica con empresas MNCS, entre I 986 y 1995, la IED en zonas francas representó, en promedio, el 20\% del total de flujos de IED hacia Costa Rica ${ }^{2}$. A inicios de la década de los noventa, las ZF empezaron a crecer significativamente, lo cual consolidó su importancia relativa en la economía costarricense. De hecho, entre 1997 y el 2007, el promedio anual de los flujos de IED de las empresas de ZF representó US\$286 millones (I,5\% del PIB, en promedio). Cabe señalar que la mayor parte de las MNCS de alta tecnología opera bajo el régimen de ZF.

Las empresas que se encuentran operando en zona franca han incrementado su contribución al producto nacional al pasar de un $0,5 \%$ a un $9,6 \%$ del $\mathrm{PIB}$, entre principios de la década de 1990 y el 2007. Las ZF no solo han incrementado directamente las oportunidades de empleo en Costa Rica, sino también las nuevas oportunidades para los trabajadores altamente cualificados. En efecto, la fuerza laboral empleada por las empresas en ZF ha crecido significativamente: desde 7000 trabajadores en 1990, a cerca de 50000 en el 2009.

Monge y González (2007) descubrieron evidencia de que algunas MNCS de alta tecnología establecidas en Costa Rica constituyen una fuente de capacitación, educación y experiencia laboral para sus empleados. Estos autores afirman que el conocimiento adquirido por empleados costarricenses en empresas como Intel, Cisco y Microsoft pareciera no ser específico de la industria en la cual estas empresas operan.

De hecho, los autores encuentran que la capacitación recibida por los empleados es del tipo de aprendizaje de por vida (lifelong learning process), dentro del cual se incluyen cursos de capacitación no solo en temas específicos para la posición que va a ocupar el empleado dentro de la MNC o para nuevos puestos que ocupará a futuro dentro

2 Estimaciones del autor con base en datos de Procomer y el Banco Central de Costa Rica (BCCR). de la misma empresa (job training plan and specific training), sino también en otras áreas definidas por el propio empleado (individual development plan) como mecanismo que le permita alcanzar sus objetivos, de acuerdo a como el empleado los perciba dentro de la empresa a mediano plazo. El tipo de capacitación descrita anteriormente incluye estudios de idiomas como inglés y portugués, comercio electrónico, planeamiento de negocios, entre otros.

\section{Movilidad laboral desde las multinacionales que operan en Costa Rica}

La movilidad laboral desde las MNCS hacia empresas locales en Costa Rica ha sido inicialmente documentada por Monge, Rosales y Arce (2005), quienes encontraron que el $36,2 \%$ de los gerentes, el $27,6 \%$ de los ingenieros y el $31 \%$ de los técnicos que trabajan en compañías locales suplidoras de insumos a MNCS han laborado previamente en MNCS en Costa Rica. Además, los autores encontraron que el 27,6\% de las empresas suplidoras locales cuentan con al menos un dueño con previa experiencia laboral en alguna de las MNCS que operan en este país.

En otro estudio, Monge (2010) analizó la movilidad laboral desde las MNCS en Costa Rica por medio de un listado de las principales MNCS que operan en el país, todas ellas atraídas por la Coalición Costarricense de Iniciativas de Desarrollo (Cinde). De acuerdo con Monge, Rivera y Rosales (20 I0), el papel de la Cinde en la atracción de flujos de IED ha sido muy significativo, pues dichos flujos representan, en promedio, el $42 \%$ del total de la IED recibida por Costa Rica durante los últimos 10 años ( 1997-2007), así como el $89 \%$ de la IED atraída bajo el régimen de ZF en el mismo periodo.

Los autores utilizaron los registros de la Caja Costarricense del Seguro Social (CCSS) sobre los empleados contratados por estas empresas, así como de aquellos que dejaron de trabajar para las MNCS entre el 2001 y el $2007^{3}$. De esta forma, fue posible identificar 4I 149 empleados de MNCS que dejaron de laborar en dichas compañías para trabajar

3 Ciertas limitaciones con respecto a la información hicieron imposible obtener los datos correspondientes a los años anteriores al 200I. Sin embargo, dado que Cinde atrajo IED en alta tecnología, principalmente a partir de la segunda mitad de la década de los noventa, el periodo estudiado puede ser considerado satisfactorio para los propósitos del presente documento. 
en otras empresas locales, o bien que dejaron de participar en el mercado laboral costarricense. Fue posible obtener el nombre, cédula de identidad, género y edad a la cual dejo de trabajar para la MNCS, de cada uno de los 4I I49 trabajadores identificados. Además, para el caso de trabajadores que se trasladaron de una MNC a una empresa local, se logró identificar el nombre de la entidad local que lo contrató y el año en que lo hizo.

De acuerdo con el estudio, 117 empresas MNCS que operan bajo el régimen de ZF en Costa Rica, principalmente en el área de alta tecnología, han empleado, en promedio, 20267 trabajadores por año, entre el 200 I y el $2007^{4}$. Por otra parte, este mismo grupo de MNCS ha experimentado una rotación de 5878 empleados promedio por año durante el mismo periodo. Esta rotación de trabajadores oscila entre un 20\% en el 2002 y un 31\% en el 2007.

Un resultado importante del estudio de Monge (2010) es el número de trabajadores que se trasladaron al sector productivo costarricense una vez que dejaron de trabajar en las MNCS. De acuerdo con el autor, entre el 200। y el 2007, de los 4I I 49 empleados de MNCS, amparadas bajo el régimen de ZF, que dejaron sus empleos, poco más de un tercio (39\%) se trasladó al sector productivo costarricense (i.e. empresas privadas, instituciones públicas, ONG, universidades, etc.) durante el periodo bajo análisis (I8 29| empleados), lo cual constituye un fuerte apoyo a la hipótesis de que las MNCS podrían estar generando derrames de conocimiento hacia el resto de la economía costarricense por medio de la movilidad laboral.

De estos 18 29| puestos de trabajo ocupados por ex empleados de MNCS en el sector productivo costarricense, casi dos terceras partes fueron ocupados por hombres (62,5\%) y una tercera parte por mujeres (37,5\%). Es importante señalar que la gran mayoría de esta población (81,7\% de los hombres y $82,5 \%$ de las mujeres) tenía menos de 35 años de edad cuando dejó de laborar para las MNCS. Es decir, que los empleados que dejaron de trabajar para MNCS amparadas bajo el régimen de ZF, lo hicieron en edades consideradas como las

4 De acuerdo con los registros de Cinde, esta institución ha atraído I55 compañías desde que fue creada en 1982. No obstante, los registros de la CCSS muestran datos de empleos para el periodo bajo estudio solo para II7 de estas empresas. más productivas. Por otro lado, la mayor parte de los empleados que se trasladaron desde una MNC al sector productivo costarricense se movió a empresas del sector privado (82,8\%); en otras palabras, I 5 I 39 de los 18 29| empleados que dejaron de laborar en MNCS se trasladaron a empresas privadas del sector productivo costarricense.

Un análisis sobre los tipos de empresas a las cuales se pasaron los ex empleados de las MNCS reveló que poco menos de tres cuartas partes de ellas (72\%) son pequeñas, medianas o grandes empresas costarricenses. Además, estas empresas absorbieron el $86 \%$ del total de ex empleados de MNCS que se trasladaron a empresas privadas entre los años 2001 y 2007.

\section{Derrames de conocimiento y desempeño de las empresas locales}

En esta sección se explora hasta qué punto la movilidad laboral desde las MNCS hacia empresas locales de Costa Rica y el derrame de conocimientos asociado a este fenómeno, han mejorado el desempeño de estas últimas en materia de ventas, empleo y productividad.

\section{Datos usados en la estimación}

Para determinar la existencia de externalidades asociadas con la movilidad laboral de ex empleados de las MNCS que operan en Costa Rica hacia empresas locales, se construyó un panel de 272 empresas con información para el periodo del 2007 al 2009. Estas empresas pertenecen a los sectores agrícola (4), explotación de minas y canteras (2), manufactura (2I2) y servicios (54). De acuerdo con el tamaño de las empresas, estas son mayoritariamente pequeñas $(59,1 \%)$, seguidas de las medianas (23,7\%), grandes $(9,2 \%)$ y microempresas $(7,9 \%)$. Es importante recordar que en las empresas locales de mayor tamaño es donde mayoritariamente se han trasladado los ex empleados de MNCS que operan en Costa Rica, tal y como se mostró al final de la sección anterior.

De la totalidad de las empresas del panel, menos de una cuarta parte de ellas ha contratado ex empleados de las MNCS entre el 2007 y el 2009 (20,4\% en el $2007 ; 24,7 \%$ en el 2008 ; y $23,9 \%$ en 
el 2009)5. Además, un porcentaje similar de las empresas manifestó que su gerente había trabajado previamente en una multinacional (23\% en el 2007; $24 \%$ en el 2008; y 25\% en el 2009). No obstante, a pesar de la similitud de los resultados anteriores, debe aclararse que solo poco más de un tercio de los gerentes que trabajaron previamente en una multinacional trabaja ahora en una empresa local que haya contratado otros ex empleados de MNCS (35\% en el 2007; 39\% en el 2008; y 37\% en el 2009). Estos resultados podrían estar señalando que el aprovechamiento de conocimientos en empresas locales donde tanto el gerente como otros empleados hayan trabajado previamente en una multinacional, es muy limitado.

Un dato interesante es el hecho de que alrededor de un 60\% de los gerentes que trabajaron previamente en MNCS manifestó haber recibido algún tipo de capacitación mientras laboró para este tipo de empresas ${ }^{6}$. Por otra parte, un porcentaje mayor (70,7\%) indicó haber desarrollado algún tipo de habilidad durante su permanencia en las MNCS. Estas habilidades son muy específicas y pocos gerentes han desarrollado el mismo tipo de habilidad.

Para poder construir el panel, fue necesario combinar tres fuentes diferentes de información: (i) fuentes secundarias, (ii) fuentes primarias y (iii) otras fuentes de información. La principal fuente secundaria la constituyen los registros de CAATEC sobre ciertas empresas locales encuestadas para otros proyectos en algún momento del 2007, 2008 o 2009. La información recopilada corresponde a datos sobre las características y la operación de las empresas.

La fuente primaria consistió en una encuesta telefónica llevada a cabo por la empresa Unimer Research International a las empresas del panel. La información recopilada mediante este procedimiento versa sobre ciertas características del gerente de las empresas, así como la contratación de ex empleados de MNCS durante los tres años incluidos en el panel. Adicionalmente, se obtuvo información sobre características propias de las empresas y de la

5 Se hace referencia a cualquier tipo de trabajador, incluyendo personal en puestos de gerencia.

6 Lamentablemente no se dispone de información sobre el tipo de capacitación recibida. No obstante, en las primeras dos secciones de este artículo se describieron algunos de los tipos de capacitación que ex empleados de MNCS, quienes posteriormente crearon sus propias empresas, recibieron durante su permanencia en una multinacional que opera en Costa Rica. actividad en la que se desempeñan. Finalmente, con el propósito de verificar cierta información relacionada con el número de trabajadores contratados por las empresas del panel, se contrastaron los datos suministrados por las compañías entrevistadas con los registros de la Caja Costarricense del Seguro Social. En caso de encontrarse alguna inconsistencia, se volvió a consultar a las empresas para corrooborar la información suministrada en primera instancia.

\section{Modelo para la estimación de externalidades}

La literatura sobre el impacto de los derrames de conocimiento asociados con la operación de MNCS o con la IED en un país anfitrión es controversial, excepto en aquellos casos en que se considera explícitamente el canal por el que se producen dichos derrames de conocimiento a las empresa locales del país anfitrión. Asimismo, debe tenerse presente que los derrames de conocimiento involucran un proceso por medio del cual las empresas locales aprenden de las empresas extranjeras. En otras palabras, es necesario tomar en consideración también la capacidad de estas empresas para absorber y adoptar nuevas tecnologías o conocimientos provenientes de las MNCS (capacidad de absorción), ya que de esto también dependerá si se producen o no efectos positivos de los derrames de conocimiento en las empresas locales (externalidades).

Knell y Rojec (20ll) señalan que, para obtener resultadosrobustosenlasestimacioneseconométricas sobre la existencia de derrames de conocimiento, es necesario tomar en cuenta los siguientes factores: primero, utilizar bases de datos panel en vez de bases de datos de corte transversal, debido a que el impacto de los derrames de conocimiento sobre el desempeño de las firmas puede tomar tiempo; segundo, controlar las diferencias en el desempeño de las empresas entre sectores que no varían en el tiempo, las cuales pueden estar correlacionadas con la operación de MNCS, pero no son causadas por su presencia en el país anfitrión (time fixed effects); tercero, utilizar variables de control que ayuden a aislar el impacto de la movilidad laboral desde las MNCS sobre el desempeño de las empresas locales, de otras variables que pueden afectar dicho desempeño; cuarto, emplear variables explicativas adicionales que tomen en cuenta la capacidad de absorción de las empresas locales, tales como su nivel de desarrollo o capacidad tecnológica (I\&D o 
innovación), el empleo de recurso humano calificado, la propensión a exportar y su nivel de productividad.

Al considerar todas las anteriores recomendaciones y la disponibilidad de información del panel construido para este estudio, se plantea el modelo usado para determinar la existencia de derrames de conocimiento por medio de la movilidad laboral desde las MNCS y su impacto sobre el desempeño de las empresas locales en Costa Rica.

El modelo es general para la empresa i en el tiempo $t$, el cual relaciona los resultados $Y_{t}$ (ventas, empleo y productividad) con una variable dicotómica $D_{t}$ que indica si la empresa contrató ex empleados de MNCS (diferentes del gerente) durante el periodo objeto de análisis; también se toman en cuenta un conjunto de atributos de la empresa observados $X_{t}$ (si el gerente trabajó en una MNCS, si recibió capacitación o desarrolló habilidades mientras laboró en la MNCS, años de escolaridad del gerente, años de laborar el gerente en el mismo sector, si la empresa innova en productos o en procesos, e importancia relativa del personal calificado), y un conjunto de variables dummies $\left(S_{j}\right.$ y $\left.T_{t}\right)$ para controlar el sector productivo $j$ al que pertenece la empresa y el tiempo, respectivamente. Esta última variable -tiempo- es importante toda vez que se trate de controlar el posible impacto de la crisis financiera mundial del 2008/2009 sobre el desempeño de la empresa.

$$
\begin{aligned}
Y_{i t} & =\mathrm{a} D_{t}+\mathrm{b} X_{t}+\mathrm{I}_{t} S_{j}+\mathrm{d}_{t} T_{t}+\mathrm{e}_{t} \\
\mathrm{e}_{i} & =\mathrm{n}_{i}+u_{t}
\end{aligned}
$$

Donde $\mathrm{e}_{i t}$ está constituido por un componente específico de la empresa $v$, invariable en el tiempo, y un término de error $u$ distribuido aleatoriamente.

\section{Estimación y resultados}

El modelo descrito en el punto anterior (I) se estima con ayuda de mínimos cuadrados ordinarios, mediante un estimador de efectos fijos y el empleo del panel de datos antes descrito. Se presentan los resultados para las tres variables de impacto $Y$ (ventas, empleo y productividad), para lo que en cada caso se utilizaron cuatro especificaciones del modelo descrito en el punto anterior. La primera especificación relaciona la variable resultado con la variable de interés $D$ (si la empresa contrató ex empleados de MNCS diferentes del gerente),
gMNCs (si el gerente trabajó previamente en una MNCS), dos variables control sobre la experiencia del gerente de la empresa (si: años de escolaridad del gerente; y eij: años de laborar el gerente en el mismo sector), así como tres variables que tratan de capturar la capacidad de absorción de la empresa local (innovapc: si la empresa innova en proceso; innovapd: si la empresa innova en producto/servicio; y prcalper: porcentaje del total de trabajadores permanentes que son profesionales o técnicos).

La segunda especificación incluye todas las variables de la primera especificación, más gcap (si el gerente recibió capacitación mientras laboró en la MNCS). Por otro lado, la tercera especificación es igual a la segunda, solo que se sustituye gcap por hab (si el gerente desarrolló habilidades mientras laboró para una MNCS). Por último, la cuarta especificación incluye todas las variables del modelo.

Cabe señalar que, en todas las anteriores especificaciones, se incluyeron interacciones (según correspondía) entre la variable $D$ y gMNCs, gcap y hab, a fin de analizar si, para la generación de externalidades, era necesario no solo contar con ex empleados de MNCS, sino también con el hecho de que estos trabajan en empresas cuyos gerentes laboraron para MNCS, o que hubieran recibido capacitación o desarrollado habilidades mientras trabajaron para este tipo de empresas; es decir, para explorar la dimensión del impacto de equipos de trabajo de ex empleados de MNCS en las empresas locales.

\section{Estimación y resultados para toda la muestra}

En el cuadro I, la primera especificación muestra un impacto positivo y significativo de la contratación de ex empleados de las MNCS en el desempeño de las empresas locales, medido por el comportamiento de sus ventas reales $(0,372)^{7}$. Es decir, la evidencia apunta hacia la existencia de una externalidad positiva de los derrames de conocimiento asociados con la movilidad laboral desde las MNCS hacia las empresas locales en Costa Rica.

Por otra parte, cuando se explora la importancia de que la empresa local sea, además, dirigida por un gerente que trabajó previamente en una MNC,

\footnotetext{
$7 \quad$ Cabe indicar que los coeficientes asociados con las dummies de tiempo y sector productivo no se presentan en el cuadro I, ya que se incluyeron en forma implícita dentro del programa de Stata empleado.
} 
Cuadro I. Costa Rica: Impacto de los derrames de conocimiento asociados con la contratación de ex empleados de MNCS sobre las ventas de las empresas locales

\begin{tabular}{|c|c|c|c|c|}
\hline \multirow{2}{*}{ Variables } & \multicolumn{4}{|c|}{ Especificaciones del modelo estimado } \\
\hline & (I) & $(2)$ & (3) & (4) \\
\hline \multirow[t]{2}{*}{$\mathrm{D}$} & $0,372 *$ & $0,380 *$ & $0,374 *$ & $0,382 *$ \\
\hline & $(0,117)$ & $(0,1 \mid 3)$ & $(0,117)$ & $(0,1 \mid 4)$ \\
\hline \multirow[t]{2}{*}{ Gmnc } & $-0,498 * * *$ & $-0,85 \mid$ I**** & $-0,200 *$ & $-0,321 * *$ \\
\hline & $(0,03 \mathrm{I})$ & $(0,035)$ & $(0,057)$ & $(0,052)$ \\
\hline \multirow[t]{2}{*}{ Dxgmnc } & 0,117 & I,096 & 0,175 & 0,935 \\
\hline & $(0,244)$ & $(0,589)$ & $(0,129)$ & $(0,495)$ \\
\hline \multirow[t]{2}{*}{ Gcap } & & $0,66 \mid$ 米米 & & I, I I 5 **** \\
\hline & & $(0,0 \mid 0)$ & & $(0,060)$ \\
\hline \multirow[t]{2}{*}{ Dxgcap } & & $-1,464$ & & $-1,961+$ \\
\hline & & $(0,534)$ & & $(0,557)$ \\
\hline \multirow[t]{2}{*}{$\mathrm{Si}$} & $0,254 * * * *$ & 0,244**** & $0,260 * * * *$ & $0,253 * * * *$ \\
\hline & $(0,002)$ & $(0,00 I)$ & $(0,003)$ & $(0,00 I)$ \\
\hline \multirow[t]{2}{*}{ Eij } & $0,008 * *$ & $0,008 * *$ & $0,008 * *$ & $0,008 * *$ \\
\hline & $(0,00 I)$ & $(0,002)$ & $(0,00 I)$ & $(0,00 I)$ \\
\hline \multirow[t]{2}{*}{ Innovapc } & 0,248 & 0,249 & 0,252 & 0,262 \\
\hline & $(0,176)$ & $(0,174)$ & $(0,178)$ & $(0,166)$ \\
\hline \multirow[t]{2}{*}{ Innovapd } & $-0,345 *$ & $-0,38 \mid *$ & $-0,308^{*}$ & $-0,335 *$ \\
\hline & $(0,098)$ & $(0,090)$ & $(0,100)$ & $(0,084)$ \\
\hline \multirow[t]{2}{*}{ Prcalper } & 0,000 & 0,000 & $-0,000$ & $-0,000$ \\
\hline & $(0,00$ I) & $(0,002)$ & $(0,00 \mathrm{I})$ & $(0,002)$ \\
\hline \multirow[t]{2}{*}{ Ghab } & & & $-0,4 \mid 3^{*} *$ & $-1,093^{*} * *$ \\
\hline & & & $(0,068)$ & $(0,107)$ \\
\hline \multirow[t]{2}{*}{ Dxghab } & & & $-0,14 \mid$ & 0,557 \\
\hline & & & $(0,255)$ & $(0,257)$ \\
\hline \multirow[t]{2}{*}{ Constant } & $|4,9| 4 * * * *$ & 15,073***** & |4,860**** & $|5,0| 4 * * * *$ \\
\hline & $(0,088)$ & $(0,108)$ & $(0,088)$ & $(0,113)$ \\
\hline Observaciones & 608 & 608 & 608 & 608 \\
\hline R-cuadrado & 0,136 & 0,144 & 0,139 & 0,152 \\
\hline Número de años & 3 & 3 & 3 & 3 \\
\hline
\end{tabular}

Nota: *Coeficiente es estadísticamente significativo al 10\%; *** al 5\%; **** al $1 \%$.

Fuente: Elaboración propia.

se obtiene un resultado significativo pero con signo negativo (-0,498). Más aún, cuando se analiza la interacción de la existencia de tal gerente con la contratación de otros ex empleados de MNCS, el resultado es nulo.

Una posible interpretación del anterior resultado es que aquellas empresas con menor grado de desempeño son las que buscan la contratación de ex empleados de MNCS para que las dirijan. Otra posibilidad es que esta variable, por sí sola, no capture el hecho de que el gerente haya incrementado su acervo de conocimientos durante su estadía en la MNCS (v.g. capacitación), los cuales emplea en la empresa local que dirige. De hecho, debe recordarse que solo un $60 \%$ de los gerentes que trabajaron previamente para MNCS manifestó haber recibido algún tipo de capacitación de parte de estas empresas.

Como era de esperarse, los años de educación y experiencia previa del gerente en el mismo sector tienen un impacto positivo y significativo en el desempeño de la empresa $(0,254$ y 0,008, respectivamente). 
Por último, de las variables que tratan de capturar la capacidad de absorción de la empresa local, solo la variable de innovación en producto (o servicio) muestra un coeficiente significativo, aunque con signo negativo $(-0,345)$. Este resultado es contra intuitivo, ya que se esperaría que empresas con mayor capacidad de innovación tuvieran un mejor desempeño en ventas. En general, podría pensarse que las empresas locales tienen muy poca capacidad de absorción, lo que les impide explotar aún más los derrames de conocimiento asociados con la movilidad laboral desde las MNCS.

Claro está, puede ser que las variables consideradas no logren capturar bien la capacidad de absorción de las empresas locales, como sí lo hacen otras especificaciones (Girma y Görg, 2002). Sin lugar a dudas, este último tema - determinar efectivamente la capacidad de absorción de las empresas- resulta de vital importancia para la definición de una política pública, sin embargo, excede los alcances de la presente investigación.

La segunda especificación del modelo agrega a los resultados anteriores la dimensión de la capacitación recibida por los gerentes mientras laboraron para alguna MNCS. Se encontró una relación positiva y significativa entre esta variable y el desempeño de la empresa local en materia de ventas $(0,661)$. Este hallazgo refuerza el impacto positivo de los derrames de conocimiento sobre las empresas locales y señala la importancia de considerar, en el análisis, el hecho de que el gerente haya sido capacitado durante su estadía en la MNC, no solamente de si el gerente trabajó en alguna empresa de este tipo antes de laborar para la empresa local.

Al explorar la dimensión del impacto de equipos de trabajo de ex empleados de MNCS en las empresas locales (interacción de D y gcap), no se encontró un impacto diferente al obtenido cuando solo el gerente proviene de una MNC, o cuando los ex empleados de MNCS laboran en una empresa local cuyo gerente no trabajó previamente en una MNC (i.e. el coeficiente asociado con gcap) ${ }^{8}$.

En la tercera especificación, los resultados no varían con respecto a las especificaciones anteriores, excepto

8 El impacto de equipos de trabajo de ex empleados de una MNC es igual a la suma de los coeficientes de gcap y Dxgcap, el cual, en este caso, es igual al coeficiente de gcap $(0,661)$ toda vez que el coeficiente asociado con Dxgcap no sea estadísticamente diferente de cero. porque se encontró una relación significativa pero negativa entre el hecho de que el gerente haya desarrollado alguna habilidad mientras laboró en la MNC y el desempeño de la empresa local que actualmente dirige. En este caso, se interpreta que quizás las habilidades desarrolladas son específicas de la industria MNC donde el gerente laboró y que, por lo tanto, estas no son necesariamente útiles en la empresa local que ahora dirige.

En la cuarta y última especificación, los resultados son muy similares a los obtenidos en las especificaciones anteriores, lo cual apoya la hipótesis de que estos son muy robustos. Es por este motivo que, en los siguientes análisis, se comentarán solo los resultados de la cuarta especificación.

El único resultado que llama la atención es el impacto negativo y significativo de los equipos de trabajo de ex empleados de MNCS en las empresas locales (interacción de D y gcap), el cual, en este caso, es de $-0,816^{9}$; sin embargo, este resultado parece contra intuitivo. Una posible interpretación es que, ante esta situación, al gerente no le es tan fácil aplicar en la empresa local aquellos conocimientos adquiridos en su capacitación durante su estadía en una MNC. En cualquier caso, se requiere de mayor investigación de campo para comprender efectivamente lo que sucede en aquellas empresas donde el gerente y algunos de sus otros trabajadores provienen de empresas MNCS.

El cuadro 2 muestra los resultados de la estimación del modelo (I) cuando la variable de resultado es el empleo y no las ventas reales de la empresa. Los resultados sobre la externalidad positiva asociada con la contratación de ex empleados de las MNCS es más robusta que en el caso de las ventas. El grado de significancia del coeficiente de la variable $D$ es mayor en las cuatro especificaciones del modelo.

La contratación de un gerente con experiencia previa en una MNC (gMNCs) y su trabajo junto a otros ex empleados de MNCS no tienen impacto alguno sobre el empleo de las empresas locales. Los coeficientes para las variables gMNCs y DxgMNCs no son significativos.

Los resultados sobre el impacto de gcap, y la interacción de Dxgcap, si y eij no varían en cuanto a dirección y significancia al compararlos con los

9 Diferencia entre el coeficiente gcap $(|| 45$,$) y Dxgcap (-1,96 \mid)$. 
Cuadro 2. Costa Rica: Impacto de los derrames de conocimiento asociados con la contratación de ex empleados de MNCS sobre el empleo de las empresas locales

\begin{tabular}{|c|c|c|c|c|}
\hline \multirow{2}{*}{ Variables } & \multicolumn{4}{|c|}{ Especificaciones del modelo estimado } \\
\hline & (I) & (2) & (3) & (4) \\
\hline \multirow[t]{2}{*}{$\mathrm{D}$} & $0,27 \mid * *$ & $0,280 * *$ & $0,266 * *$ & $0,272 * *$ \\
\hline & $(0,050)$ & $(0,047)$ & $(0,049)$ & $(0,048)$ \\
\hline \multirow[t]{2}{*}{ Gmnc } & 0,045 & $-0,182 * *$ & 0,151 & 0,090 \\
\hline & $(0,074)$ & $(0,039)$ & $(0,059)$ & $(0,057)$ \\
\hline \multirow[t]{2}{*}{ Dxgmnc } & 0,060 & $0,918 * *$ & $-0,142$ & 0,530 \\
\hline & $(0,032)$ & $(0,125)$ & $(0,095)$ & $(0,184)$ \\
\hline \multirow[t]{2}{*}{ gcap } & & $0,44 \mid *$ & & $0,708 * *$ \\
\hline & & $(0,104)$ & & $(0,092)$ \\
\hline \multirow[t]{2}{*}{ Dxgcap } & & $-1,227 * *$ & & $-1,535 * *$ \\
\hline & & $(0,218)$ & & $(0,205)$ \\
\hline \multirow[t]{2}{*}{$\mathrm{Si}$} & $0,109 * * *$ & 0,103 ***** & $0,11 \mid * * * *$ & $0,106 * * *$ \\
\hline & $(0,00 \mathrm{I})$ & $(0,002)$ & $(0,00 \mathrm{I})$ & $(0,002)$ \\
\hline \multirow[t]{2}{*}{ eij } & $0,005 * *$ & $0,006 * *$ & 0,005 *** & $0,005 * *$ \\
\hline & $(0,00 I)$ & $(0,00 I)$ & $(0,00 I)$ & $(0,001)$ \\
\hline \multirow[t]{2}{*}{ innovapc } & $0,237 *$ & $0,220 *$ & $0,241 *$ & $0,227 *$ \\
\hline & $(0,063)$ & $(0,073)$ & $(0,058)$ & $(0,067)$ \\
\hline \multirow[t]{2}{*}{ innovapd } & 0,006 & $-0,001$ & 0,006 & 0,003 \\
\hline & $(0,0 \mid 0)$ & $(0,009)$ & $(0,0 \mid I)$ & $(0,006)$ \\
\hline \multirow[t]{2}{*}{ prcalper } & $-0,006 * *$ & $-0,006 * *$ & $-0,006 * *$ & $-0,006 * *$ \\
\hline & $(0,00 I)$ & $(0,00 I)$ & $(0,00 I)$ & $(0,00 \mathrm{I})$ \\
\hline \multirow[t]{2}{*}{ ghab } & & & $-0,149$ & $-0,57 \mid * * *$ \\
\hline & & & $(0,087)$ & $(0,062)$ \\
\hline \multirow[t]{2}{*}{ Dxghab } & & & 0,295 & $0,804 * *$ \\
\hline & & & $(0,174)$ & $(0,105)$ \\
\hline \multirow[t]{2}{*}{ Constant } & 1,387 ***** & $|, 51|$ I**** & 1,347**** & I,431**** \\
\hline & $(0,033)$ & $(0,048)$ & $(0,0 \mid 4)$ & $(0,03 \mathrm{I})$ \\
\hline Observaciones & 784 & 784 & 784 & 784 \\
\hline R-cuadrado & 0,114 & 0,127 & 0,115 & 0,134 \\
\hline Número de años & 3 & 3 & 3 & 3 \\
\hline
\end{tabular}

Nota: *Coeficiente es estadísticamente significativo al 10\%, ** al 5\%, *** al $1 \%$.

Fuente: Elaboración propia.

obtenidos en la estimación del modelo (I), cuando las ventas de la empresa constituyen la variable dependiente.

Dos nuevos e importantes resultados se obtienen en la cuarta estimación del modelo (I) cuando la variable resultado es el empleo. Primero, aquellas empresas que innovan en procesos (innovapc) tienen un mejor desempeño en materia de generación de empleos, lo cual pareciera apoyar la tesis de que la capacidad de adopción de las empresas locales es una variable importante a considerar en el análisis de los derrames de conocimiento. Segundo, el trabajo conjunto de ex gerentes que hayan desarrollado habilidades mientras trabajaron en MNCS y ex empleados de estas empresas que ahora laboran para la empresa local, resulta en un impacto positivo sobre la generación de empleos en estas últimas (i.e. suma de los coeficientes ghab y Dxghab en la cuarta especificación) $)^{10}$.

Finalmente, cabe señalar que no se encontró externalidades asociadas con la contratación de ex empleados de MNCS por parte de las empresas locales cuando la variable de resultado es la productividad media del trabajo (valor agregado

10 Es decir, $-0,57 \mid+0,804=0,233$, ambos coeficientes significativos. 
por trabajador). Es decir, no se identificó impacto alguno de este hecho sobre la productividad de las empresas locales. Debido a limitaciones de espacio no se presentan los resultados de este modelo.

Como corolario de esta sección, se puede concluir que, al tomar los resultados en su conjunto, estos presentan una evidencia consistente de que las empresas locales que han contratado ex empleados de MNCS muestran un mejor desempeño en términos de crecimiento de ventas y empleo, con respecto a sus contrapartes locales. Es decir, estos resultados constituyen una clara evidencia de una externalidad positiva asociada con los derrames de conocimiento productos de la movilidad laboral desde las MNCS hacia las empresas locales de Costa Rica. Además, pareciera importante llevar a cabo nuevas investigaciones cuyos resultados ayuden a la definición de políticas públicas que mejoren la capacidad de absorción de las empresas locales.

\section{Consideraciones finales}

A manera de conclusión general, se puede afirmar que existe evidencia consistente sobre importantes externalidades positivas asociadas con los derrames de conocimiento producto de la movilidad laboral desde las MNCS hacia las empresas locales en Costa Rica.

Si bien se identificaron externalidades positivas de la contratación de ex empleados de MNCS por parte de empresas locales, aún hay espacio para mejorar la capacidad de absorción de estas empresas para admitir y adaptar tecnologías y nuevos conocimientos que mejoren su desempeño. Esfuerzos en esta dirección incluyen la mejora del recurso humano de las empresas locales, su capacidad de innovación, su vocación exportadora y su incorporación a cadenas globales de valor en procesos productivos de empresas exportadoras. En esta misma línea, es importante reforzar la estrategia de promoción de IED, incluyendo dentro de esta estrategia la promoción de IED asociada con el área de investigación y desarrollo.

Finalmente, los esfuerzos por mejorar el nivel educativo de la fuerza laboral costarricense deben establecerse como una constante, toda vez que de ello dependa, en buena medida, la capacidad de los empleados de MNCS para absorber conocimientos que posteriormente podrán utilizar en sus propias empresas o en otras empresas locales que operen en Costa Rica.

En futuros trabajos de investigación, sería interesante profundizar acerca del impacto de los derrames de conocimiento sobre la productividad de las empresas creadas por ex empleados de MNCS, midiendo dicha productividad en términos de productividad total de los factores y no como productividad media del trabajo. Finalmente, también es de interés analizar la capacidad de absorción de las empresas que hayan contratado ex empleados de MNCS en comparación con otras empresas.

\section{Bibliografía}

Blomström, M. (1986). Foreign investment and productive efficiency: the case of Mexico. Journal of Industrial Economics, 35(I): 97-1 10.

Buckley, P.; Clegg, J. \& Wang, C. (2007). Is the relationship between inward FDI and spillover effects linear? An empirical examination of the case of China. Journal of International Business Studies, 38(3): 447-459.

Caves, R. (1974). Multinational firms, competition and productivity in host-country markets. Economica, 4I (162): 176-193.

Feinberg, S. \& Majumdar, S. (200I). Technology spillovers from foreign direct investment in the Indian pharmaceutical industry. Journal of International Business Studies, 32(3): 421-437.

Girma, S. \& H. Görg. (2002). Foreign Direct Investment, Spillovers and Absorption Capacity: Evidence from Quantile Regressions. GEP Research Paper 02/14, University of Nottingham, Nottingham.

Görg, H. \& Strobl, E. (200I). Multinational companies and productivity spillovers: a meta-analysis. Economic Journal. I | I: 723-739.

Görg, H. \& Strobl, E. (2005). Spillovers from foreign firms through worker mobility: An empirical investigation, Scandinavian Journal of Economics, 107(4): 693-709.

Hale, G. \& Long, C. (2006). What Determines Technological Spillovers of Foreign Direct Investment? Evidence from China. Yale Economic Growth Center Discussion Paper 934, New Haven, CT.

Jovanovic, B. (1982). Selection and the Evolution of Industry. Econometrica, 50 (3): 649-70.

Knell, M. \& Rojec, M. (20I I). Why is there little evidence of knowledge spillovers from foreign direct investment? Unpublished academic paper. 
Lim, E. (200 I). Determinants of, and the Relation Between Foreign Direct Investment and Growth:A Summary of the Recent Literature. IMFWorking PaperWP/0 I/I 75.

Markusen, J. \& Trofimenko, N. (2007). Teaching Locals New Tricks: Foreign Experts as a Channel of Knowledge Transfer. NBER Working Paper 12872. Cambridge: National Bureau of Economic Research.

Monge, R. (20 I 0).Transmisión de conocimientos en Costa Rica. En O. Céspedes y A. Pacheco. (Eds.). Ensayos en Honor a Cecilia Valverde Barrenechea. San José: Costa Rica: Academia de Centroamérica.

Monge, R. \& Lizano, E. (1997). Apertura Económica e Industrialización en Costa Rica. San José: Academia de Centroamérica.

Monge, R.; Rosales, J. \& Arce, G. (2005). Análisis costobeneficio del régimen de zonas francas: Impactos de la inversión extranjera directa en Costa Rica. Office of Commerce, Growth and Competitiveness, Organization of American States.

Monge, R. \& González, C. (2007). The role and impact of MNCS in Costa Rica on skills development and training: The case of Intel, Microsoft and Cisco. Geneva: The International Labor Organization.
Monge, R.; Rivera, L. \& Rosales, J. (2010). Productive Development Policies in Costa Rica: Market Failures, Government Failures and Policy Outcomes. Working Paper Series No. IDB-WP-157. Washington D.C.: Inter-American Development Bank.

Poole, J. (2008). Multinational spillovers through worker turnover. Department of Economics, University of California, Santa Cruz.

Saggi, K. (2002). Trade, foreign direct investment and international technology transfer: A survey. World Bank Research Observer, 17(2): 191-235.

Spencer, J. (2008). The impact of multinational enterprise strategy on indigenous enterprises: horizontal spillovers and crowding out in developing countries. Academy of Management Review, 33:341-36 I.

Tian, X. (2007). Accounting for sources of FDI technology spillovers: evidence from China. Journal of International Business Studies, 38: I47- 159.

Wei, Y. \& Liu, X. (2006). Productivity spillovers from R\&D, exports and FDI in China's manufacturing sector. Journal of International Business Studies, 37(4): 544-557.

Zhang,Y.; Li, H.; Li,Y. \& Zhou, L. (20 I 0). FDI Spillovers in an emerging market: The role of foreign firm's country origin diversity and domestic firm's absorptive capacity. Strategic Management Journal, 31: 969-89. 